\title{
Adipose tissue and age-dependent insulin resistance: New insights into WAT browning (Review)
}

\author{
CHUANLONG WU ${ }^{1 *}$, PEI YU ${ }^{1 *}$ and RUIXIN SUN ${ }^{2}$ \\ ${ }^{1}$ Department of Orthopaedics, Shanghai Key Laboratory for Prevention and Treatment of Bone and Joint Diseases, \\ Shanghai Institute of Traumatology and Orthopaedics, Ruijin Hospital, Shanghai Jiao Tong University School of Medicine, \\ Shanghai 200025; ${ }^{2}$ State Key Laboratory of Oncogenes and Related Genes, Shanghai Cancer Institute, \\ Renji Hospital, Shanghai Jiao Tong University School of Medicine, Shanghai 200032, P.R. China
}

Received November 8, 2020; Accepted February 3, 2021

DOI: $10.3892 /$ ijmm.2021.4904

\begin{abstract}
Insulin resistance (IR) is defined as impaired insulin function, reduced glucose uptake and increased glucose production, which can result in type II diabetes, metabolic syndrome and even bone metabolic disorders. A possible reason for the increasing incidence of IR is population aging. Adipose tissue (AT) is an important endocrine organ that serves a crucial role in whole-body energy homeostasis. AT can be divided into white AT (WAT), beige AT and brown AT (BAT). Several mechanisms have been previously associated with age-dependent IR in WAT. However, BAT, a metabolically active tissue, controls the levels of plasma glucose and triglyceride metabolism. Therefore, the present review aimed to summarize the mechanisms of age-dependent IR induced by AT and to determine the role of WAT browning in achieving positive therapeutic outcomes in age-dependent IR.
\end{abstract}

Correspondence to: $\mathrm{Dr}$ Chuanlong $\mathrm{Wu}$, Department of Orthopaedics, Shanghai Key Laboratory for Prevention and Treatment of Bone and Joint Diseases, Shanghai Institute of Traumatology and Orthopaedics, Ruijin Hospital, Shanghai Jiao Tong University School of Medicine, 197 Ruijin 2nd Road, Shanghai 200025, P. R. China

E-mail: challengewu1988@163.com

Professor Ruixin Sun, State Key Laboratory of Oncogenes and Related Genes, Shanghai Cancer Institute, Renji Hospital, Shanghai Jiao Tong University School of Medicine, Lane 2200, 25 Xietu Road, Shanghai 200032, P.R. China

E-mail: qingshuiqiuqian@126.com

*Contributed equally

Key words: insulin resistance, aging, white adipose tissue, brown adipose tissue, type II diabetes

\section{Contents}

1. Introduction

2. AT characteristics

3. WAT in age-dependent IR

4. BAT in age-dependent IR

5. Beige AT in age-dependent IR

6. Therapeutic value of beige adipocytes and acquisition of brown-like properties in WAT

7. Conclusion

\section{Introduction}

Insulin resistance (IR) is defined as impaired insulin function, reduced glucose uptake and increased glucose production (1), which may result in type II diabetes and metabolic syndrome (2) and may subsequently promote the development of non-alcoholic fatty liver disease (NAFLD) (3). Numerous previous studies (4-6) have reported mechanisms through which IR serves a crucial role in a variety of systemic diseases, including bone metabolic disorders (7). The etiology of IR is complex and a possible reason for the increasing incidence of IR is the aging population, as epidemiological research has reported that the prevalence of IR and type II diabetes is higher in older adults. It has also been demonstrated that aging induces IR, and in turn, hyperinsulinemia and IR may accelerate aging, promoting a vicious circle $(8,9)$.

Adipose tissue (AT) is an active organ regulating ATP expenditure, which serves an essential role in regulating energy homeostasis of the entire body (10). Our previous studies focusing on the function of AT identified that the dysfunction of AT induced IR $(11,12)$ and more recent studies $(13,14)$ have also reported a crucial role for AT in IR during aging. Therefore, an improved understanding of the effects of aging on AT may prove useful in pioneering novel therapeutic strategies to target age-related diseases. The present review aimed to summarize the associative evidence between AT and age-dependent IR. Notably, the findings suggested that the browning of white AT (WAT) may be influenced to attain positive therapeutic outcomes in age-dependent IR and other metabolic complications. 


\section{AT characteristics}

AT can be subdivided into three subtypes, namely WAT, brown AT (BAT) and beige AT, which all possess distinct characteristic features that are associated with their function, localization and composition (15). BAT develops following the stimulation of anatomical thermogenic sites corresponding with WAT in a process termed 'browning' $(15,16)$. In mammals, BAT and WAT have opposing functions. For example, BAT expends energy, while WAT stores energy. Significant changes are known to occur over the years in the distribution and composition of AT during aging. The progressive dysfunction of AT has been proposed to be a characteristic feature of the aging process (17).

White adipocytes. The majority of AT in the human body is WAT, which is distinguishable by large unilocular lipid droplets. Functionally, WAT is subdivided into a stromal vascular fraction, which is composed of lymphocytes, progenitor and endothelial cells, preadipocytes and fibroblasts, and the adipocyte fraction, which primarily contains mature adipocytes $(17,18)$. WAT is dispersed throughout visceral organs and the surrounding subcutaneous region of the face. Notably, despite their histological similarities, visceral AT (VAT) and subcutaneous tissue have been shown to exert distinct metabolic functions. Subcutaneous WAT has metabolic characteristics that differ from those of VAT. For example, the metabolic activity of WAT is regulated by a smaller number of insulin receptors, $\beta$-adrenoceptors ( $\beta$-AR) and glucocorticoid receptors (19).

Brown adipocytes. BAT is a major source of metabolically active fat and is located in the retroperitoneal, cervical and mediastinal regions (20). BAT is named due to the brown color of the adipocytes and has a high mitochondria content and vascular supply. BAT can arise from precursor cells already present in WAT, which display identical unilocular morphology as WAT during the basal state (21). However, the morphological characteristic features of BAT are altered upon cold stimulation, eventually resulting in the expression of typical BAT proteins, and the transformation from stored fat to small lipid droplets, which are typical of BAT (22). The beta-3 adrenergic receptor ( $\beta 3-\mathrm{AR}$ ), type 2 iodothyronine deiodinase and uncoupling protein 1 (UCP1) are highly expressed in the adipocytes of BAT (23), in which UCP1 functions as a thermoregulator (24).

Beige adipocytes. Beige adipocytes are brown-like adipocytes that exist within WAT in adult humans and play an essential role in ATP expenditure (25), which are characterized by a mix of the features of both brown and white adipocytes (21). Inactive beige cells appear morphologically as regular white adipocytes, although upon stimulation (through adrenergic receptors), oxidative and mitochondrial biogenesis is enhanced (25). However, it has been suggested that beige adipocytes may serve a role similar to the role brown adipocytes play in the expenditure of ATP, which has been demonstrated in humans (26). Notably, beige adipocytes have also been found to be transplantable. Several studies have suggested that beige stem cells may derive from WAT cells by trans-differentiation, which is driven by bone morphogenetic protein $7(21,27)$. However, there is also evidence to suggest that beige adipocytes may arise from unique precursor cells (25). For instance, Wu et al (25) reported that beige adipocytes were differentiated from myogenic factor 5 (Myf5) ${ }^{+}$progenitor cells. Nevertheless, an ongoing debate still surrounds the anatomical location, trans-differentiation and cellular identity of the three adipocyte subtypes.

\section{WAT in age-dependent IR}

It has been reported that the accumulation of senescent cells was increased in the WAT of diabetic patients (28); thus, the association between WAT and aging may be of clinical significance, as numerous classical aging mechanisms have been shown to occur in WAT, which may be associated with to age-dependent IR (29).

Adipocyte self-renewal. In adults, human WAT is dynamic and can differentiate into mature lipid-storing adipocytes. In total, $\sim 10 \%$ of adipocytes die and renew every year (30). Renewed progenitor cells were demonstrated to play a crucial role in insulin sensitivity (IS), the expansion of AT and lipid handling. Thus, the failure of progenitor cells to self-renew could lead to the exhaustion of new adipocytes, resulting in the senescence and death of adipocytes (31).

It is estimated that $15-50 \%$ of cells in WAT are preadipocytes that are continuously renewed throughout an individual's life, which results in the varying size and functional changes observed in older adults compared with younger individuals $(32,33)$. Thus, determining the association between fat mass expansion and adipogenesis may be essential for establishing the pathophysiological role that adipocyte turnover plays in IR. Notably, the function of progenitor cells, such as those within the skeletal muscle, also decreases with age (age-related IR). Thus, the inherent characteristics of progenitor cells may contribute to the variation in WAT function with aging $(34,35)$.

The reduced ability of adipocytes to differentiate during aging may combine with the decreased insulin responsiveness of WAT when exposed to excess nutrients during old age. Although very little is known regarding the precise age, percentage and required threshold, progenitor cells may cause physiological changes or become dysfunctional (36).

Location of AT. The location of AT is equally as essential as the absolute amount of AT during reduced IS. Fat redistribution in the elderly has been associated with an increased risk of IS (37). Subcutaneous WAT and VAT are diverse with regards to their metabolic effects; for example, subcutaneous WAT has been associated with increased IS and a lower risk of cardiovascular disease (CVD) and diabetes, while VAT has been associated with IR (38). Furthermore, pear-shaped fat distribution with more gluteal femoral AT was associated with an increased IS and lower risk of CVD and diabetes, while apple-shaped fat distribution with an increased waist circumference was associated with VAT mass and IR. Individuals with relatively higher amounts of VAT compared with WAT were also at an increased risk of developing IR (39). The loss of subcutaneous fat is a well-established feature in aging (38), 
as the distribution of AT primarily moves from subcutaneous fatty tissue deposition to ectopic sites, muscle and visceral deposition, including within the liver, which are all closely associated with the development of IR (40).

Increased VAT accumulation was demonstrated to result in lipotoxicity in old age (>65 years old) (41) and chronic low-grade inflammation (42). The central accumulation of VAT during aging has been closely associated with an increase of inflammatory and metabolic markers compared with peripheral fat accumulation (43). Similar to humans, the redistribution of fat in rat and mice models tended to occur with aging due to insulin-like growth factor-1 and/or growth hormone deficiencies (44). As reported by Niu et al (45), diabetes and IR was effectively reduced in aging rats by antagonizing the age-dependent accumulation of VAT.

Volume of adipocytes. Body fat mass typically increases by either hypertrophy or hyperplasia, with the former involving slower rates of adipogenesis compared with the latter. Hypertrophic adipocytes were reported to induce hypoxic conditions and have been implicated in several types of condition, including atherosclerosis, type II diabetes and IR. The development of hypoxia underpins the infiltration of macrophages, which subsequently promotes the initiation and progression of the inflammatory response in AT (46).

The migration of macrophages results in impaired insulin signaling, which is known to be central to the development of IR and metabolic syndrome (Fig. 1). In addition, during hypertrophy, adipocytes present in the senescent state and secrete a multitude of growth factors, cytokines, matrix metalloproteinases and chemokines (47). Thus, removing senescent cells in progeroid INK-ATTAC mice has been found to improve WAT IS $(48,49)$. Free fatty acids (FFAs) are essential biomedical indicators of lipid metabolic disorders, whereby the effects of FFAs on IR are dose-dependent, which adversely affects insulin signaling pathways (45). Plasma FFA levels have been associated with aging and excess FFA accumulation was found to induce IR during aging (50).

An increase in FFAs has demonstrated to induce IR at the cellular level, while increasing very-low-density hepatic lipoprotein production (44). It has also been reported that the accumulation of FFAs, triglycerides (TGs) and diglycerides may promote adipocyte necrosis in obese mice, which resulted in the polarization of macrophages towards a standard M1 or activated phenotype in the AT.

MicroRNA (miRNA) processing. miRNAs have been identified in multiple tissues, including the skeletal muscles, AT, liver and brain (51). The number of miRNAs present in WAT was found to decrease with age, owing to the downregulation of the miRNA-processing enzyme known as Dicer15 (52). An experimental study with mice demonstrated that Dicer15 exhibited a defect in miRNA processing in AT, prevented the whitening of BAT and resulting in an inadequate response of WAT to metabolic stress, and promoted lipodystrophy, IR and inflammation (53). In addition, AT was recently reported to be an essential source of circulating exosomal miRNAs, which have been associated with glucose tolerance; thus, representing a novel target to produce therapeutics that modulate age-related metabolic diseases and various aging processes (54).
Adipokines. WAT secretes varying amounts of bioactive molecules named adipokines, which serve crucial roles in regulating diverse metabolic processes ranging from food intake to nutrient recycling, energy homeostasis and IR (55). It has been hypothesized that age-associated changes may, however, influence the impact of adipokine secretion (56).

Adiponectin. Adiponectin is an adipose-derived hormone that has been associated with an improved IS, which also inhibits the synthesis of proinflammatory cytokines (57). As adipokines secreted from WAT are involved in the regulation of glucose metabolism, it has been suggested that adiponectin may mediate IS and possess anti-inflammatory properties (58). However, the association between aging and adiponectin remains poorly understood; for example, several previous studies have reported that plasma adiponectin levels increased with age $(59,60)$, while other studies reported the opposite results (61). Overall, the relative decrease in adiponectin secretion in VAT has been associated with an increased risk of aging (62).

In addition, lower levels of adiponectin have been associated with obesity, cigarette smoking and oxidative stress. One of the most prominent hypotheses regarding the mechanisms inducing or worsening obesity and consequently, NAFLD, is the overproduction of reactive oxygen species (ROS) (63).

Leptin. Leptin is a WAT-secreted hormone, which stimulates fatty acid oxidation and regulates insulin binding and production, by interacting with regions of the brain involved in controlling appetite (64). In addition, the availability of leptin was found to influence lipogenesis and lipolysis, and the failure of leptin to restore metabolic homeostasis is termed as a state of leptin resistance (65). Notably, aging has been associated with a decrease in leptin activity, while youth is associated with a high sensitivity of leptins; The redistribution of adipose tissue observed during old age contribute however to an increase in circulating leptin. However, leptin resistance could not be overcome by an increase in leptin levels during aging. This suggested that leptin resistance appears to be an early contributor to the development of metabolic abnormalities in old age $(64,66)$.

Resistin. Resistin is activated during the process of IR (insulin antagonism) and is a distinctive cysteine-rich signaling molecule. In mice, resistin is produced by monocytes and AT, while in humans, resistin is produced in monocytes and macrophages, but not in AT (67). Circulating resistin levels have been reported to increase with age, and elevated levels of resistin were associated with an increased risk for coronary intervention and CVDs (68).

Inflammation. Low-grade, chronic inflammation, which is a central mechanism involved in the aging process, has been established as a precursor of aging and closely associated with IR (69). The hypotheses surrounding the WAT inflammatory capacity suggest that immune cells infiltrating WAT are the primary source of inflammation (70). Adipose resident immune cells, such as M2 macrophages, which are anti-inflammatory cells, have been reported to improve IS, whereas M1 macrophages, mast cells, natural killer cells and neutrophils, which are proinflammatory cells, promote IR during aging (71,72). Therefore, immune cells and their participation in the inflammatory response are important pathophysiological mechanisms of aging WAT (Fig. 2). 


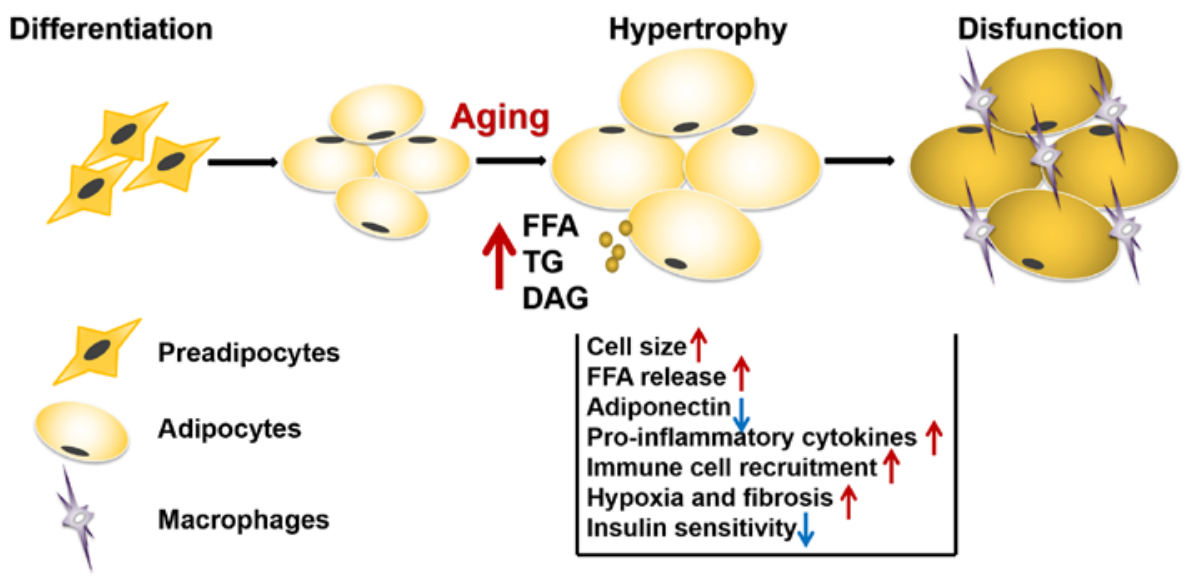

Figure 1. Adipocyte hypertrophy results in a slower adipogenic ability and cell dysfunction compared with adipocyte hyperplasia. The average fat cell volume increases with age. Moreover, the accumulation of FFAs, TGs and DAGs in WAT induces the infiltration of macrophages, leading to impaired insulin signaling and the dysfunction of adipocytes, ultimately resulting in IR. FFAs, free fatty acids; TGs, triglycerides; DAGs, diglycerides; IR, insulin resistance; WAT, white adipose tissue.

AT macrophages (ATMs). ATMs are induced by aging and secrete chemokines that attract monocytes to the AT. ATMs consist of two subsets: Proinflammatory M1 ATMs (which secrete TNF- $\alpha$ ) and anti-inflammatory M2 ATMs (which secrete arginase 1) (73). Proinflammatory ATMs also secrete their own chemokines once they migrate into the WAT, attracting additional macrophages and promoting the inflammatory processes (74).

AT T cells. T cells differentiate into proinflammatory phenotypes with elevated levels of chemokine receptors and enhanced chemotaxis potential. Notably, aging has been found to alter $\mathrm{T}$ cell development. $\mathrm{T}$ regulatory $\left(\mathrm{T}_{\text {reg }}\right)$ cells, a small subset of $\mathrm{T}$ cells, are an essential defense mechanism of the body against inappropriate immune responses due to inflammation, autoimmunity, infection, cancer or allergies. Recently, $\mathrm{T}_{\text {reg }}$ cells were identified to be involved in controlling the inflammatory state of the AT. At birth, subcutaneous AT and VAT contain low percentages of $\mathrm{T}_{\mathrm{reg}}$ cells; however, over time, the percentage of $\mathrm{T}_{\text {reg }}$ cells increases in the VAT (75).

Adipose-secreted inflammatory cytokines. Aging has also been associated with higher levels of proinflammatory cytokines, which act in a paracrine or an autocrine manner to induce IR by decreasing the expression of insulin receptor substrate (IRS)-1 (76). For example, TNF- $\alpha$, expressed primarily by AT macrophages, induced IR and inhibited insulin signaling in human adipocytes by affecting the expression of IRS proteins (77). A previous study has also shown that inflammatory responses disrupt normal lipid accumulation. For example, proinflammatory cytokines, such as IL-6, impaired lipid accumulation by promoting Wnt signaling (78).

Mitochondrial dysfunction. Cumulative molecular damage can result in mitochondrial impairment (78). The mitochondria are the primary origin of ROS, which are inevitable by-products of oxidative phosphorylation. Mitochondrial dysfunction and oxidative stress in WAT are important factors leading to IR during the aging process. Mitochondrial ROS were demonstrated to attenuate insulin action in adipocytes and abolish insulin-stimulated glucose transporter 4 translocation in mouse adipocytes. Notably, impaired mitochondrial function was also shown to increase endoplasmic reticulum

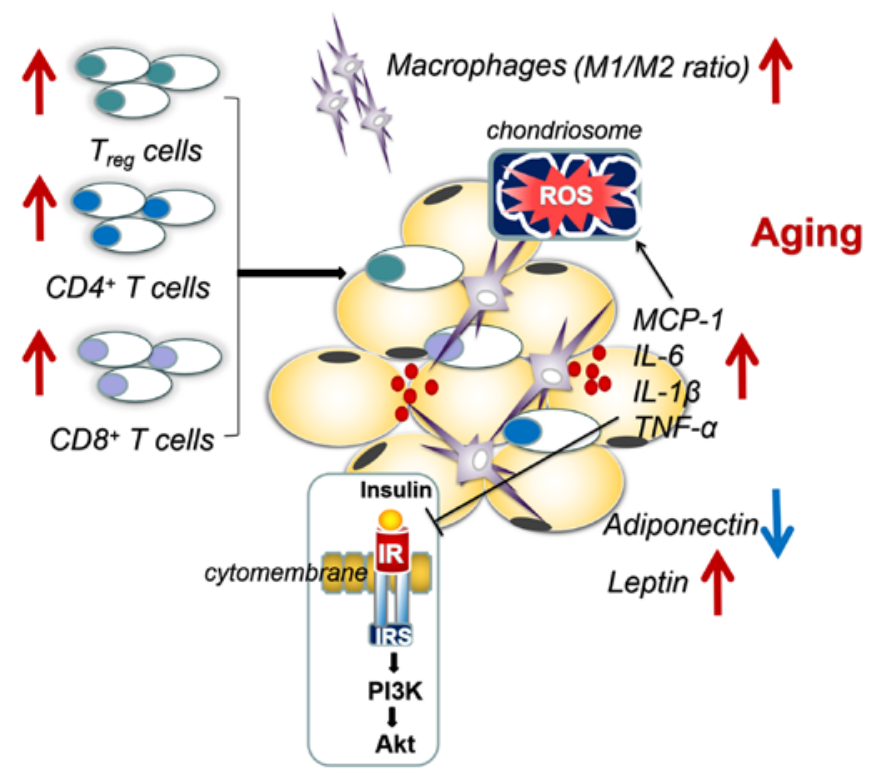

Figure 2. Chronic inflammation is closely associated with age-dependent IR. The expression levels of inflammatory factors, such as MCP-1, IL-6, IL-1, TNF- $\alpha$ and leptin are upregulated, while the expression levels of adiponectin are downregulated in aging WAT. Subsequently, aging WAT is infiltrated by macrophages, $\mathrm{CD}^{+} \mathrm{T}$ cells, $\mathrm{CD} 8^{+} \mathrm{T}$ cells and $\mathrm{T}_{\text {reg }}$ cells. Excessive metabolic products flux into the mitochondria during aging, resulting in the overproduction of ROS and spin-down of electrons, which can result in oxidative stress and ultimately, damage the activation of the PI3K/AKT signaling pathway, thereby inducing IR. MCP-1, monocyte chemotactic protein 1; WAT, white adipose tissue; $\mathrm{T}_{\text {reg }}, \mathrm{T}$ regulatory; ROS, reactive oxygen species; IR, insulin resistance; IRS, insulin receptor substrate.

stress $(78,79)$. Mitochondria also serve a central role in cell death; therefore, mitochondrial dysfunction in adipocytes may trigger cell death in adipocytes to induce AT inflammation (79). Thus, the dysfunction of mitochondria highly probably a significant cause of age-dependent chronic inflammation.

Mitochondrial sirtuins (mtSIRTs) comprise three members, SIRT3, SIRT4 and SIRT5, which are all involved in regulating energy metabolism and metabolic homeostasis (80). Among the mtSIRTs, SIRT4 expression levels were found to be upregulated during senescence and were induced by different stimuli, 
such as DNA damage (80). SIRT4 functions as an efficient mitochondrial ADP-ribosyl transferase that inhibits mitochondrial glutamate dehydrogenase (GDH) activity. GDH is known to convert glutamate to $\alpha$-ketoglutarate, which promotes ATP generation and insulin secretion in pancreatic $\beta$ cells (81). In vivo experiments observed a significant increase in insulin secretion from SIRT4-deficient pancreatic $\beta$ cells in response to glucose and amino acids in SIRT4-knockout (KO) mice. More recently, SIRT4 has also been shown to interact with insulin-degrading enzyme, which modulates insulin secretion, providing an alternative possible mechanism by which SIRT4 may impact insulin secretion (82).

\section{BAT in age-dependent IR}

BAT is a metabolically active tissue that regulates plasma glucose levels, IR, TG metabolism and hyperlipidemia (83). Notably, it has recently emerged as a potential target in the treatment of type II diabetes. BAT has the capacity to actively clear glucose from the circulation and has been suggested to serve a role in preventing age-dependent dysfunction and disease (83).

Abnormalities in BAT. A notable change in AT distribution associated with aging is due to the loss of BAT. A decline in BAT function with aging has been described in both rodents and humans (84). In one study, the proportion of BAT was $>50 \%$ in younger individuals ( $<35$ years old), but $<10 \%$ in older individuals ( $>65$ years old) (84). In addition, metabolic alterations in subcutaneous WAT harbored a subpopulation of $\mathrm{UCP}^{+}$brown adipocytes, thereby promoting IR (85). Indeed, a predominant consequence of aging in murine subcutaneous WAT is the loss of 'browning' subcutaneous WAT, and UCP1 expression in subcutaneous WAT was found to be increasingly downregulated with age in rodents (85). Loss of BAT in older mice was also inversely correlated with IR development (86). Furthermore, it has been suggested that BAT itself may become dysfunctional with age (86).

Mitochondrial mass in BAT. In addition to TG stores in multilocular lipid droplets, BAT has high mitochondrial density. During aging, there is a significant decline of UCP1 activity and an age-dependent accumulation of point mutations in mitochondrial DNA (87). Cumulative molecular damage was found to result in mitochondrial functional decline and impairment (88), which are both associated with an age-dependent increase in the incidence of neurodegenerative diseases and metabolic disorders (89).

Inflammation in BAT. As aforementioned, chronic inflammation increases with age. It was previously demonstrated that BAT is resistant to macrophage infiltration, as shown by the downregulated expression of immune cell-enriched genes, which indicates the anti-inflammatory properties of BAT (90). However, during the inflammatory response in mice, a novel chemokine (C-X-C chemokine ligand 14) was observed to be secreted by thermogenically activated BAT (91). In addition, several proinflammatory cytokines reduce UCP1 gene expression in BAT. For instance, TNF- $\alpha$ induces the apoptosis of WAT and downregulates the expression of UCP1 via Toll-like receptor activation, thereby decreasing thermogenesis in BAT (92).

Hormonal regulation of BAT function. The age-dependent decline of BAT activity has been suggested to be associated with changes in circulating levels of hormones. Ghrelin signaling is an essential thermogenic regulator during aging (93). Obestatin, which upregulates UCP1 expression, and Ghrelin, which downregulates UCP1 expression, are both derived from the same pre-proghrelin gene. During aging, following no change in plasma obestatin expression, increased growth hormone secretagogue receptor (GHS-R) expression and plasma ghrelin expression in BAT was demonstrated to promote thermogenic regulatory imbalance in middle-aged and old mice. Moreover, the knockdown of the ghrelin receptor and/or GHS-R decreased the risk of age-associated IR in Ghsr/mice (94). Thyroid hormones, such as triiodothyronine, were also demonstrated to be regulators of thermogenesis; therefore, a decrease in thyroid hormones may significantly contribute to the dysfunction of BAT during aging (95).

\section{Beige AT in age-dependent IR}

Numerous molecular mechanisms have been reported to be involved in the browning of WAT during aging. The origin of beige adipocytes may result from a white-to-brown trans-differentiation and/or the de novo differentiation of specific precursor cells. For example, progenitor cells and/or adipose stem cells of WAT may differentiate into white or beige adipocytes (96). Among them, a distinct subpopulation of WAT resident progenitors expressing CD137 and transmembrane protein 26 (TMEM26) are more able to differentiate into beige adipocytes (25). Thus, the age-related lose of beige AT may be explained by the loss of CD137/TMEM $26^{+}$progenitors. In addition, several regulators were demonstrated to serve important roles in the browning of WAT. For example, peroxisome proliferator activated receptor (PPAR) $\gamma$ and PR/SET domain 16 (PRDM16) are not only be involved in the induction of BAT-associated genes, but also in the repression of WAT-associated genes. PRDM16 upregulated the expression levels of BAT-specific genes by associating with the transcriptional co-activators, PPAR $\gamma$ coactivator 1 (PGC)-1 $\alpha$ and PGC-1 $\beta$ (97). SIRT1, an important target in adipose biology, facilitated the browning of AT via PPAR $\gamma$ and PRDM16 (98) and enhanced beige adipocyte differentiation ability via the senescence-associated p53/p21 signaling pathway (99), which is downregulated during aging. Irisin was also reported to upregulate the expression of UCP1 and promote the WAT browning process, which is a novel hormonal factor that converts WAT into BAT (100). Notably, irisin also induced a significant increase in total body energy expenditure (101). In conclusion, the combined effect of the aforementioned factors have been suggested to participate in the browning process.

\section{Therapeutic value of beige adipocytes and acquisition of brown-like properties in WAT}

In a previous study, BAT transplantation in mice improved whole-body energy expenditure and IS, suggesting a key role of WAT browning in improving whole body energy metabolism 


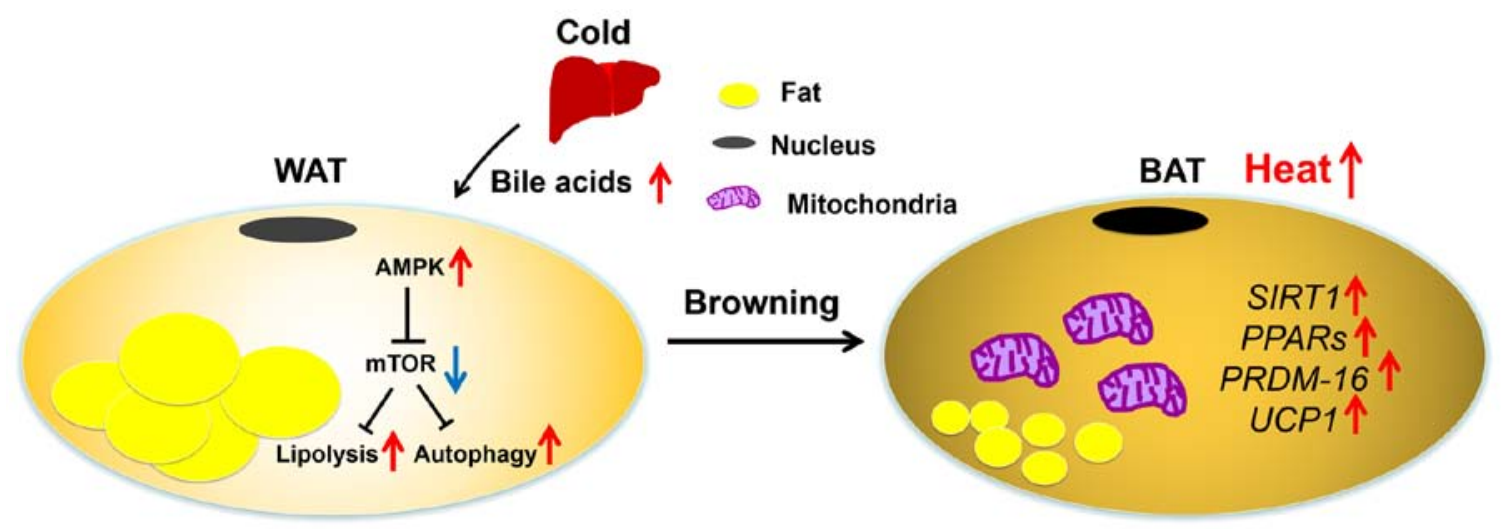

Figure 3. Possible mechanisms involved in the process of WAT browning. Under cold exposure, thermogenic brown and beige adipocytes produce heat by oxidizing fatty acids. Liver-derived lipid metabolites and bile acids stimulate thermogenesis in BAT. With cold exposure, the levels of bile acid were elevated. $\beta$-adrenergic receptors are activated by the release of noradrenaline, and the resultant $\mathrm{Ca}^{2+}$ influx causes AMPK-dependent SIRT1 activation, which deacetylates PPAR $\gamma$ and PRDM-16. A PPAR $\gamma /$ PRDM-16 interaction results in the browning of inguinal WAT. WAT, white adipose tissue; BAT, brown adipose tissue; AMPK, 5'AMP-activated protein kinase; SIRT1, sirtuin 1; PPAR $\gamma$, peroxisome proliferator activated receptor $\gamma$; PRDM-16, PR/SET domain family-16; UCP-1, uncoupling protein 1; SIRT1, sirtuin-1.

and IS (102). Thus, it may be important to shift beige adipose cells from a WAT phenotype to a BAT phenotype under specific stimuli (Fig. 3).

Cold exposure. Cold exposure is a strong beige adipocyte inducer. Significant metabolism and gene expression changes in WAT were found to be induced by chronic cold exposure (103). For example, PGC-1 $\alpha$ expression levels, a co-activator of PPAR $\gamma$, were upregulated upon cold exposure. In primary human subcutaneous WAT, the overexpression of PGC-1 $\alpha$ promoted polarization to a BAT phenotype, which was accompanied by the upregulated expression levels of fatty acid oxidation enzymes, respiratory chain proteins and UCP1 (104). In addition, chronic cold exposure was also observed to stimulate the differentiation of precursors into beige adipocytes within one week of exposure (105).

Physical exercise. Physical exercise has been reported to be associated with a reduction in lipid content, increased mitochondrial activity and the modified secretion of adipokines (106). However, the effects of physical exercise in the AT of the elderly, particularly in BAT, remain unknown. To date, the findings of previous studies investigating the effect of exercise on BAT in the elderly remain controversial. Recent studies revealed that endurance exercise training could facilitate brown-like adipocyte recruitment within WAT by activating cytokines such as irisin and IL-6 in rodents (107). Furthermore, strength and aerobic training induced an increase in BAT mitochondrial activity in old rats (108). However, another study revealed that chronic endurance exercise was not associated with the recruitment of brown and beige adipocytes (109). Thus, further studies are required to investigate the complex association between different types of exercise and exercise-induced WAT and BAT adaptations.

Diet. Previous studies have reported that several food ingredients may stimulate thermogenesis and browning of the WAT. For example, in a human model, a significant increase in insulin-induced fluorodeoxyglucose uptake in BAT was observed, suggesting that BAT may influence postprandial energy metabolism (110). In addition, capsaicin and capsinoids, which are alkaloids extracted from Capsicum genus, simulated the heat burning sensation (111). The chronic oral administration of capsinoids each day also triggered a significant increase in BAT activity (111). Fucoxanthin (FX), which was found to be present in abundance in the diatom Phaeodactylum tricornutum, significantly downregulated the expression levels and activity of lipogenic enzymes, upregulated the expression levels of AR- $\beta 3$ in adipocytes and the fatty acid oxidation rate (112). In both WAT and BAT, the administration of a high-fat diet mixed with $0.2 \% \mathrm{FX}$ upregulated the expression levels of UCP1 in mice. Carotenoids have a large spectrum of isoprenoids, such as $\beta$-carotene and retinoic acid, which regulate thermogenesis and energy expenditure in both WAT and BAT (113). $\beta$-carotene modulates the expression levels of UCP1 and retinoic acid upregulates the expression levels of catabolic and thermogenic proteins that increase mitochondrial biogenesis. Long-chain $\omega-3$ polyunsaturated fatty acids (LCPUFAs), in particular eicosapentaenoic acid (EPA), upregulated the expression levels of mitochondrial oxidative-and thermogenic-associated genes, in addition to increasing 5'AMP-activated protein kinase (AMPK) phosphorylation and carnitine palmitoyltransferase 1 expression in mice, suggesting that the treatment with EPA may improve energy expenditure, thermogenesis and oxidation in subcutaneous adipocytes (114). Polyphenols, which are secondary metabolites of plants, were also found to recruit new brown adipocytes, and increase metabolic activity, including WAT browning (115).

Autophagy. Autophagy, a cell-protective dynamic process, rearranges subcellular membranes to segregate the cytoplasm and organelles for transmission to lysosomes, and promotes cell death via the excessive degradation of cellular constituents, which may have a role in regulating the browning of WAT. After knocking down the expression of autophagy-related 7 (ATG7) in $\mathrm{aP}^{+}$adipocytes to impair autophagy, a BAT phenotype was evidenced by an increased WAT and BAT mass (116). Concurrently, brown adipocyte differentiation and function were specifically disrupted by suppressing ATG7 in Myf5 ${ }^{+}$ 
precursor cells (117). In addition, mTOR is an essential negative regulator of autophagy. Previous studies have shown that the inhibition of mTOR blocked the cold-induced browning of WAT (118). The $\beta$-AR-dependent upregulation of UCP1 expression and increase in the number of beige adipocytes in WAT were observed in mice with mTOR complex 1 impairment (119). Another previous study suggested that the tumor suppressor folliculin (FLCN) regulated WAT browning via a transcription factor binding the IGHM enhancer 3 (TFE3) and mTOR. mTOR-dependent cytoplasmic retention of TFE3 was relieved by the adipose-specific deletion of FLCN, resulting in the activation of the PGC- $1 \alpha$ transcriptional co-activator function, suggesting that adipose browning was suppressed by mTOR (120).

\section{Conclusion}

As there are multiple different mechanisms to induce the WAT browning process, it is plausible that the browning process may represent a potential target to achieve efficacious therapeutic outcomes in aging-dependent IR. Numerous molecules that induce BAT activation or WAT browning may represent potential targets to treat various disease types associated with IR, including type II diabetes, metabolic syndrome and bone diseases.

\section{Acknowledgements}

Not applicable.

\section{Funding}

The present study was supported by grants from the National Natural Science Foundation of China (grant no. 81802169) and Shanghai Sailing Program (grant no. 18YF1413900).

\section{Availability of data and materials}

Not applicable.

\section{Authors' contributions}

RS designed the concept of the review and its structure. CW wrote and revised the manuscript. PY was involved in the writing of the review. All authors agree to be accountable for all aspects of the work in ensuring that questions related to the accuracy or integrity of any part of the work are appropriately investigated and resolved.

\section{Ethics approval and consent to participate}

Not applicable.

\section{Patient consent for publication}

Not applicable.

\section{Competing interests}

The authors declare that they have no competing interests.

\section{References}

1. Ikemura M, Nishikawa M, Hyoudou K, Kobayashi Y, Yamashita F and Hashida M: Improvement of insulin resistance by removal of systemic hydrogen peroxide by PEGylated catalase in obese mice. Mol Pharm 7: 2069-2076, 2010.

2. Guo S: Insulin signaling, resistance, and the metabolic syndrome Insights from mouse models into disease mechanisms. J Endocrinol 220: T1-T23, 2014.

3. Tarantino G, Citro V and Capone D: Nonalcoholic fatty liver disease: A challenge from mechanisms to therapy. J Clin Med 9: 15, 2019.

4. Czech MP: Insulin action and resistance in obesity and type 2 diabetes. Nat Med 23: 804-814, 2017.

5. Qi X, Yun C, Sun L, Xia J, Wu Q, Wang Y, Wang L, Zhang Y, Liang X, Wang L, et al: Gut microbiota-bile acid-interleukin-22 axis orchestrates polycystic ovary syndrome. Nat Med 25: 1225-1233, 2019.

6. Petersen MC and Shulman GI: Mechanisms of insulin action and insulin resistance. Physiol Rev 98: 2133-2223, 2018.

7. Napoli N, Chandran M, Pierroz DD, Abrahamsen B, Schwartz AV and Ferrari SL; IOF Bone and Diabetes Working Group: Mechanisms of diabetes mellitus-induced bone fragility. Nat Rev Endocrinol 13: 208-219, 2017.

8. Chow HM, Shi M, Cheng A, Gao Y, Chen G, Song X, So RWL, Zhang $\mathbf{J}$ and Herrup K: Age-related hyperinsulinemia leads to insulin resistance in neurons and cell-cycle-induced senescence. Nat Neurosci 22: 1806-1819, 2019.

9. Barzilai $\mathrm{N}$ and Ferrucci L: Insulin resistance and aging: A cause or a protective response? J Gerontol A Biol Sci Med Sci 67: 1329-1331, 2012.

10. Rosen ED and Spiegelman BM: Adipocytes as regulators of energy balance and glucose homeostasis. Nature 444: 847-853, 2006.

11. Sun R, Wu Y, Hou W, Sun Z, Wang Y, Wei H, Mo W and Yu M: Bromodomain-containing protein 2 induces insulin resistance via the mTOR/Akt signaling pathway and an inflammatory response in adipose tissue. Cell Signal 30: 92-103, 2017.

12. Zong J, Li S, Wang Y, Mo W, Sun R and Yu M: Bromodomaincontaining protein 2 promotes lipolysis via ERK/HSL signalling pathway in white adipose tissue of mice. Gen Comp Endocrinol 281: 105-116, 2019.

13. Bapat SP, Myoung Suh J, Fang S, Liu S, Zhang Y, Cheng A, Zhou C, Liang Y, LeBlanc M, Liddle C, et al: Depletion of fat-resident Treg cells prevents age-associated insulin resistance. Nature 528: 137-141, 2015.

14. Sierra Rojas JX, Garcia-San Frutos M, Horrillo D, Lauzurica N, Oliveros E, Carrascosa JM, Fernández-Agulló T and Ros M: Differential development of inflammation and insulin resistance in different adipose tissue depots along aging in wistar rats: Effects of caloric restriction. J Gerontol A Biol Sci Med Sci 71: 310-322, 2016.

15. Park A, Kim WK and Bae KH: Distinction of white, beige and brown adipocytes derived from mesenchymal stem cells. World J Stem Cells 6: 33-42, 2014.

16. Giralt $\mathrm{M}$ and Villarroya F: White, brown, beige/brite: Different adipose cells for different functions? Endocrinology 154: 2992-3000, 2013.

17. Palmer AK and Kirkland JL: Aging and adipose tissue: Potential interventions for diabetes and regenerative medicine. Exp Gerontol 86: 97-105, 2016.

18. Madonna R and De Caterina R: In vitro neovasculogenic potential of resident adipose tissue precursors. Am J Physiol Cell Physiol 295: C1271-1280, 2008.

19. Chon SH and Pappas A: Differentiation and characterization of human facial subcutaneous adipocytes. Adipocyte 4: 13-21, 2015.

20. Frontini A and Cinti S: Distribution and development of brown adipocytes in the murine and human adipose organ. Cell Metab 11: 253-256, 2010.

21. Cedikova M, Kripnerová M, Dvorakova J, Pitule P, Grundmanova M, Babuska V, Mullerova D and Kuncova J: Mitochondria in white, brown, and beige adipocytes. Stem Cells Int 2016: 6067349, 2016.

22. Ye L, Wu J, Cohen P, Kazak L, Khandekar MJ, Jedrychowski MP, Zeng X, Gygi SP and Spiegelman BM: Fat cells directly sense temperature to activate thermogenesis. Proc Natl Acad Sci USA 110: 12480-12485, 2013.

23. Cypess AM, White AP, Vernochet C, Schulz TJ, Xue R, Sass CA, Huang TL, Roberts-Toler C, Weiner LS, Sze C, et al: Anatomical localization, gene expression profiling and functional characterization of adult human neck brown fat. Nat Med 19: 635-639, 2013. 
24. Mailloux RJ and Harper ME: Uncoupling proteins and the control of mitochondrial reactive oxygen species production. Free Radic Biol Med 51: 1106-1115, 2011.

25. Wu J, Boström P, Sparks LM, Ye L, Choi JH, Giang AH, Khandekar M, Virtanen KA, Nuutila P, Schaart G, et al: Beige adipocytes are a distinct type of thermogenic fat cell in mouse and human. Cell 150: 366-376, 2012.

26. Pyrzak B, Demkow U and Kucharska AM: Brown adipose tissue and browning agents: Irisin and FGF21 in the development of obesity in children and adolescents. Adv Exp Med Biol 866: 25-34, 2015

27. Okla M, Ha JH, Temel RE and Chung S: BMP7 drives human adipogenic stem cells into metabolically active beige adipocytes. Lipids 50: 111-120, 2015.

28. Palmer AK, Tchkonia T, LeBrasseur NK, Chini EN, Xu M and Kirkland JL: Cellular senescence in type 2 diabetes: A therapeutic opportunity. Diabetes 64: 2289-2298, 2015.

29. Zoico E, Rubele S, De Caro A, Nori N, Mazzali G, Fantin F Rossi A and Zamboni M: Brown and beige adipose tissue and aging. Front Endocrinol (Lausanne) 10: 368, 2019.

30. Spalding KL, Arner E, Westermark PO, Bernard S, Buchholz BA, Bergmann O, Blomqvist L, Hoffstedt J, Näslund E, Britton T, et al Dynamics of fat cell turnover in humans. Nature 453: 783-787, 2008.

31. Eckel-Mahan K, Ribas Latre A and Kolonin MG: Adipose stromal cell expansion and exhaustion: Mechanisms and consequences. Cells 9: 863, 2020.

32. Tchkonia T, Morbeck DE, Von Zglinicki T, Van Deursen J, Lustgarten J, Scrable H, Khosla S, Jensen MD and Kirkland JL: Fat tissue, aging, and cellular senescence. Aging Cell 9: 667-684, 2010.

33. Caso G, McNurlan MA, Mileva I, Zemlyak A, Mynarcik DC and Gelato MC: Peripheral fat loss and decline in adipogenesis in older humans. Metabolism 62: 337-340, 2013.

34. Bukowska J, Frazier T, Smith S, Brown T, Bender R, McCarthy M, Wu X, Bunnell BA and Gimble JM: Bone marrow adipocyte developmental origin and biology. Curr Osteoporos Rep 16: 312-319, 2018.

35. Ahmed AS, Sheng MH, Wasnik S, Baylink DJ and Lau KW: Effect of aging on stem cells. World J Exp Med 7: 1-10, 2017.

36. Kirkland JL, Tchkonia T, Pirtskhalava T, Han J and Karagiannides I: Adipogenesis and aging: Does aging make fat go MAD? Exp Gerontol 37: 757-767, 2002.

37. Gill LE, Bartels SJ and Batsis JA: Weight management in older adults. Curr Obes Rep 4: 379-388, 2015.

38. Sepe A, Tchkonia T, Thomou T, Zamboni M and Kirkland JL: Aging and regional differences in fat cell progenitors-a mini-review. Gerontology 57: 66-75, 2011.

39. Pischon T, Boeing H, Hoffmann K, Bergmann M, Schulze MB, Overvad K, van der Schouw YT, Spencer E, Moons KG, Tjønneland A, et al: General and abdominal adiposity and risk of death in Europe. N Engl J Med 359: 2105-2120, 2008.

40. Preis SR, Massaro JM, Robins SJ, Hoffmann U, Vasan RS, Irlbeck T, Meigs JB, Sutherland P, D'Agostino RB Sr, O'Donnell CJ and Fox CS: Abdominal subcutaneous and visceral adipose tissue and insulin resistance in the Framingham heart study. Obesity (Silver Spring) 18: 2191-2198, 2010.

41. Paradis ME, Hogue MO, Mauger JF, Couillard C, Couture P, Bergeron $\mathrm{N}$ and Lamarche B: Visceral adipose tissue accumulation, secretory phospholipase A2-IIA and atherogenecity of LDL. Int J Obes (Lond) 30: 1615-1622, 2006.

42. Giorgino F: Adipose tissue function and dysfunction: Organ cross talk and metabolic risk. Am J Physiol Endocrinol Metab 297: E975-E976, 2009.

43. Capurso $\mathrm{C}$ and Capurso A: From excess adiposity to insulin resistance: The role of free fatty acids. Vascul Pharmacol 57: 91-97, 2012.

44. Ibrahim MM: Subcutaneous and visceral adipose tissue: Structural and functional differences. Obes Rev 11: 11-18, 2010.

45. Niu Z, Lin N, Gu R, Sun Y and Feng Y: Associations between insulin resistance, free fatty acids, and oocyte quality in polycystic ovary syndrome during in vitro fertilization. J Clin Endocrinol Metab 99: E2269-E2276, 2014.

46. Chait A and den Hartigh LJ: Adipose tissue distribution, inflammation and its metabolic consequences, including diabetes and cardiovascular disease. Front Cardiovasc Med 7: 22, 2020.

47. Coppé JP, Patil CK, Rodier F, Sun Y, Muñoz DP, Goldstein J, Nelson PS, Desprez PY and Campisi J: Senescence-associated secretory phenotypes reveal cell-nonautonomous functions of oncogenic RAS and the p53 tumor suppressor. PLoS Biol 6: 2853-2868, 2008.
48. Xu M, Palmer AK, Ding H, Weivoda MM, Pirtskhalava T, White TA, Sepe A, Johnson KO, Stout MB, Giorgadze N, et al: Targeting senescent cells enhances adipogenesis and metabolic function in old age. Elife 4: e12997, 2015.

49. Xu M, Tchkonia T, Ding H, Ogrodnik M, Lubbers ER, Pirtskhalava T, White TA, Johnson KO, Stout MB, Mezera V, et al: JAK inhibition alleviates the cellular senescence-associated secretory phenotype and frailty in old age. Proc Natl Acad Sci USA 112: E6301-E6310, 2015.

50. Park SS and Seo YK: Excess accumulation of lipid impairs insulin sensitivity in skeletal muscle. Int J Mol Sci 21: 1949, 2020.

51. Pincus Z, Smith-Vikos T and Slack FJ: MicroRNA predictors of longevity in caenorhabditis elegans. PLoS Genet 7: e1002306, 2011.

52. Mori MA, Raghavan P, Thomou T, Boucher J, Robida-Stubbs S, Macotela Y, Russell SJ, Kirkland JL, Blackwell TK and Kahn CR: Role of microRNA processing in adipose tissue in stress defense and longevity. Cell Metab 16: 336-347, 2012.

53. Mori MA, Thomou T, Boucher J, Lee KY, Lallukka S, Kim JK, Torriani M, Yki-Järvinen H, Grinspoon SK, Cypess AM and Kahn CR: Altered miRNA processing disrupts brown/white adipocyte determination and associates with lipodystrophy. J Clin Invest 124: 3339-3351, 2014.

54. Mori MA, Ludwig RG, Garcia-Martin R, Brandão BB and Kahn CR: Extracellular miRNAs: From biomarkers to mediators of physiology and disease. Cell Metab 30: 656-673, 2019.

55. Fontes-Carvalho R, Fontes-Oliveira M, Sampaio F, Mancio J, Bettencourt N, Teixeira M, Rocha Gonçalves F, Gama V and Leite-Moreira A: Influence of epicardial and visceral fat on left ventricular diastolic and systolic functions in patients after myocardial infarction. Am J Cardiol 114: 1663-1669, 2014.

56. Mancuso $P$ and Bouchard B: The impact of aging on adipose function and adipokine synthesis. Front Endocrinol (Lausanne) 10: 137, 2019

57. Chandrasekar B, Boylston WH, Venkatachalam K, Webster NJ, Prabhu SD and Valente AJ: Adiponectin blocks interleukin-18-mediated endothelial cell death via APPL1-dependent AMP-activated protein kinase (AMPK) activation and IKK/NF-kappaB/PTEN suppression. J Biol Chem 283: 24889-24898, 2008.

58. Jura M and Kozak LP: Obesity and related consequences to ageing. Age (Dordr) 38: 23, 2016.

59. Koh KK, Quon MJ, Han SH, Lee Y, Ahn JY, Kim SJ, Koh Y and Shin EK: Simvastatin improves flow-mediated dilation but reduces adiponectin levels and insulin sensitivity in hypercholesterolemic patients. Diabetes Care 31: 776-782, 2008.

60. Isobe T, Saitoh S, Takagi S, Takeuchi H, Chiba Y, Katoh N and Shimamoto K: Influence of gender, age and renal function on plasma adiponectin level: The Tanno and Sobetsu study. Eur J Endocrinol 153: 91-98, 2005.

61. Takenouchi Y, Kobayashi T, Matsumoto T and Kamata K: Gender differences in age-related endothelial function in the murine aorta. Atherosclerosis 206: 397-404, 2009.

62. Li JB, Nishida M, Kaimoto K, Asakawa A, Chaolu H, Cheng KC, Li YX, Terashi M, Koyama KI, Amitani H, et al: Effects of aging on the plasma levels of nesfatin- 1 and adiponectin. Biomed Rep 2: 152-156, 2014.

63. Nigro E, Scudiero O, Monaco ML, Palmieri A, Mazzarella G, Costagliola C, Bianco A and Daniele A: New insight into adiponectin role in obesity and obesity-related diseases. Biomed Res Int 2014: 658913, 2014.

64. Carter S, Caron A, Richard D and Picard F: Role of leptin resistance in the development of obesity in older patients. Clin Interv Aging 8: 829-844, 2013.

65. Katsiki N, Mikhailidis DP and Banach M: Leptin, cardiovascular diseases and type 2 diabetes mellitus. Acta Pharmacol Sin 39: 1176-1188, 2018.

66. Doherty GH: Obesity and the ageing brain: Could leptin play a role in neurodegeneration? Curr Gerontol Geriatr Res 2011: 708154, 2011

67. Lehrke M, Reilly MP, Millington SC, Iqbal N, Rader DJ and Lazar MA: An inflammatory cascade leading to hyperresistinemia in humans. PLoS Med 1: e45, 2004.

68. Gencer B, Auer R, de Rekeneire N, Butler J, Kalogeropoulos A, Bauer DC, Kritchevsky SB, Miljkovic I, Vittinghoff E, Harris T and Rodondi N: Association between resistin levels and cardiovascular disease events in older adults: The health, aging and body composition study. Atherosclerosis 245: 181-186, 2016.

69. Rea IM, Gibson DS, McGilligan V, McNerlan SE, Alexander HD and Ross OA: Age and age-related diseases: Role of inflammation triggers and cytokines. Front Immunol 9: 586, 2018. 
70. de Heredia FP, Gómez-Martinez S and Marcos A: Obesity, inflammation and the immune system. Proc Nutr Soc 71: 332-338, 2012.

71. Lumeng CN, Liu J, Geletka L, Delaney C, Delproposto J, Desai A, Oatmen K, Martinez-Santibanez G, Julius A, Garg S and Yung RL: Aging is associated with an increase in T cells and inflammatory macrophages in visceral adipose tissue. J Immunol 187: 6208-6216, 2011.

72. Lumeng CN, Bodzin JL and Saltiel AR: Obesity induces a phenotypic switch in adipose tissue macrophage polarization. J Clin Invest 117: 175-184, 2007.

73. Russo L and Lumeng CN: Properties and functions of adipose tissue macrophages in obesity. Immunology 155: 407-417, 2018

74. Olefsky JM and Glass CK: Macrophages, inflammation, and insulin resistance. Annu Rev Physiol 72: 219-246, 2010.

75. Fang W, Deng Z, Benadjaoud F, Yang D, Yang C and Shi GP: Regulatory $T$ cells promote adipocyte beiging in subcutaneous adipose tissue. FASEB J 34: 9755-9770, 2020.

76. Schenk S, Saberi M and Olefsky JM: Insulin sensitivity: Modulation by nutrients and inflammation. J Clin Invest 118 2992-3002, 2008.

77. Lumeng CN, Deyoung SM and Saltiel AR: Macrophages block insulin action in adipocytes by altering expression of signaling and glucose transport proteins. Am J Physiol Endocrinol Metab 292: E166-E174, 2007.

78. Gustafson B and Smith U: Cytokines promote Wnt signaling and inflammation and impair the normal differentiation and lipid accumulation in 3T3-L1 preadipocytes. J Biol Chem 281: 9507-9516, 2006

79. Woo CY, Jang JE, Lee SE, Koh EH and Lee KU: Mitochondrial dysfunction in adipocytes as a primary cause of adipose tissue inflammation. Diabetes Metab J 43: 247-256, 2019.

80. van de Ven RAH, Santos D and Haigis MC: Mitochondrial sirtuins and molecular mechanisms of aging. Trends Mol Med 23: 320-331, 2017

81. Tao Y, Huang C, Huang Y, Hong L, Wang H, Zhou Z and Qiu Y: SIRT4 suppresses inflammatory responses in human umbilical vein endothelial cells. Cardiovasc Toxicol 15: 217-223, 2015.

82. Argmann C and Auwerx J: Insulin secretion: SIRT4 gets in on the act. Cell 126: 837-839, 2006.

83. Bartelt A, Bruns OT, Reimer R, Hohenberg H, Ittrich H, Peldschus K, Kaul MG, Tromsdorf UI, Weller H, Waurisch C, et al: Brown adipose tissue activity controls triglyceride clearance. Nat Med 17: 200-205, 2011.

84. Yoneshiro T, Aita S, Matsushita M, Okamatsu-Ogura Y, Kameya T, Kawai Y, Miyagawa M, Tsujisaki M and Saito $M$ : Age-related decrease in cold-activated brown adipose tissue and accumulation of body fat in healthy humans. Obesity (Silver Spring) 19: 1755-1760, 2011

85. Tan CY, Virtue S, Bidault G, Dale M, Hagen R, Griffin JL and Vidal-Puig A: Brown adipose tissue thermogenic capacity is regulated by Elovl6. Cell Rep 13: 2039-2047, 2015.

86. Rogers NH, Landa A, Park S and Smith RG: Aging leads to a programmed loss of brown adipocytes in murine subcutaneous white adipose tissue. Aging Cell 11: 1074-1083, 2012

87. Valle A, Guevara R, Garcia-Palmer FJ, Roca P and Oliver J: Caloric restriction retards the age-related decline in mitochondrial function of brown adipose tissue. Rejuvenation Res 11: 597-604, 2008

88. Detmer SA and Chan DC: Functions and dysfunctions of mitochondrial dynamics. Nat Rev Mol Cell Biol 8: 870-879, 2007.

89. Lin AL, Coman D, Jiang L, Rothman DL and Hyder F: Caloric restriction impedes age-related decline of mitochondrial function and neuronal activity. J Cereb Blood Flow Metab 34: 1440-1443, 2014

90. Fitzgibbons TP, Kogan S, Aouadi M,Hendricks GM, Straubhaar J and Czech MP: Similarity of mouse perivascular and brown adipose tissues and their resistance to diet-induced inflammation. Am J Physiol Heart Circ Physiol 301: H1425-H1437, 2011.

91. Villarroya F, Cereijo R, Villarroya J, Gavaldà-Navarro A and Giralt M: Toward an understanding of how immune cells control brown and beige adipobiology. Cell Metab 27: 954-961, 2018.

92. Lorenzo M, Fernández-Veledo S, Vila-Bedmar R, GarciaGuerra L, De Alvaro C and Nieto-Vazquez I: Insulin resistance induced by tumor necrosis factor-alpha in myocytes and brown adipocytes. J Anim Sci 86: E94-E104, 2008.

93. Amitani M, Amitani H, Cheng KC, Kairupan TS, Sameshima N, Shimoshikiryo I, Mizuma K, Rokot NT, Nerome Y, Owaki T, et al: The role of ghrelin and ghrelin signaling in aging. Int $\mathbf{J}$ Mol Sci 18: 1511, 2017
94.Lee JY, Takahashi N, Yasubuchi M, Kim YI, Hashizaki H, Kim MJ, Sakamoto T, Goto T and Kawada T: Triiodothyronine induces UCP-1 expression and mitochondrial biogenesis in human adipocytes. Am J Physiol Cell Physiol 302: C463-C472, 2012.

95. Weiner J, Hankir M, Heiker JT, Fenske W and Krause K Thyroid hormones and browning of adipose tissue. Mol Cell Endocrinol 458: 156-159, 2017.

96. Gustafson B, Hedjazifar S, Gogg S, Hammarstedt A and Smith U: Insulin resistance and impaired adipogenesis. Trends Endocrinol Metab 26: 193-200, 2015.

97. Kajimura S, Seale P, Tomaru T, Erdjument-Bromage $\mathrm{H}$ Cooper MP, Ruas JL, Chin S, Tempst P, Lazar MA and Spiegelman BM: Regulation of the brown and white fat gene programs through a PRDM16/CtBP transcriptional complex. Genes Dev 22: 1397-1409, 2008.

98. Becerril S, Gómez-Ambrosi J, Martin M, Moncada R, Sesma P, Burrell MA and Frühbeck G: Role of PRDM16 in the activation of brown fat programming. Relevance to the development of obesity. Histol Histopathol 28: 1411-1425, 2013.

99. Khanh VC, Zulkifli AF, Tokunaga C, Yamashita T, Hiramatsu Y and Ohneda O: Aging impairs beige adipocyte differentiation of mesenchymal stem cells via the reduced expression of Sirtuin 1. Biochem Biophys Res Commun 500: 682-690, 2018.

100. Boström P, Wu J, Jedrychowski MP, Korde A, Ye L, Lo JC Rasbach KA, Boström EA, Choi JH, Long JZ, et al: A PGC1- $\alpha$-dependent myokine that drives brown-fat-like development of white fat and thermogenesis. Nature 481: 463-468, 2012

101. Niranjan SB, Belwalkar SV, Tambe S, Venkataraman K and Mookhtiar KA: Recombinant irisin induces weight loss in high fat DIO mice through increase in energy consumption and thermogenesis. Biochem Biophys Res Commun 519: 422-429, 2019.

102. Shankar K, Kumar D, Gupta S, Varshney S, Rajan S, Srivastava A, Gupta A, Gupta AP, Vishwakarma AL, Gayen JR and Gaikwad AN: Role of brown adipose tissue in modulating adipose tissue inflammation and insulin resistance in high-fat diet fed mice. Eur J Pharmacol 854: 354-364, 2019.

103. Wang W and Seale P: Control of brown and beige fat development. Nat Rev Mol Cell Biol 17: 691-702, 2016.

104. He L, Tang M, Xiao T, Liu H, Liu W, Li G, Zhang F, Xiao Y, Zhou Z, Liu F and Hu F: Obesity-associated miR-199a/214 cluster inhibits adipose browning via PRDM16-PGC-1 $\alpha$ transcriptional network. Diabetes 67: 2585-2600, 2018.

105. Yao L, Cui X, Chen Q, Yang X, Fang F, Zhang J, Liu G, Jin W and Chang Y: Cold-inducible SIRT6 regulates thermogenesis of brown and beige fat. Cell Rep 20: 641-654, 2017.

106. Gollisch KS, Brandauer J, Jessen N, Toyoda T, Nayer A, Hirshman MF and Goodyear LJ: Effects of exercise training on subcutaneous and visceral adipose tissue in normal- and high-fat diet-fed rats. Am J Physiol Endocrinol Metab 297: E495-E504, 2009.

107. Knudsen JG, Murholm M, Carey AL, Biensø RS, Basse AL, Allen TL, Hidalgo J, Kingwell BA, Febbraio MA, Hansen JB and Pilegaard H: Role of IL- 6 in exercise training- and cold-induced UCP1 expression in subcutaneous white adipose tissue. PLoS One 9: e84910, 2014.

108. Thirupathi A, da Silva Pieri BL, Queiroz JAMP, Rodrigues MS, de Bem Silveira G, de Souza DR, Luciano TF, Silveira PCL and De Souza CT: Strength training and aerobic exercise alter mitochondrial parameters in brown adipose tissue and equally reduce body adiposity in aged rats. J Physiol Biochem 75 : 101-108, 2019.

109. Vosselman MJ, Hoeks J, Brans B, Pallubinsky H, Nascimento EB, van der Lans AA, Broeders EP, Mottaghy FM, Schrauwen P and van Marken Lichtenbelt WD: Low brown adipose tissue activity in endurance-trained compared with lean sedentary men. Int J Obes (Lond) 39: 1696-1702, 2015.

110. Orava J, Nuutila P, Lidell ME, Oikonen V, Noponen T, Viljanen T, Scheinin M, Taittonen M, Niemi T, Enerbäck S and Virtanen KA: Different metabolic responses of human brown adipose tissue to activation by cold and insulin. Cell Metab 14: 272-279, 2011

111. Nirengi $\mathrm{S}$, Homma $\mathrm{T}$, Inoue $\mathrm{N}$, Sato $\mathrm{H}$, Yoneshiro $\mathrm{T}$, Matsushita M, Kameya T, Sugie H, Tsuzaki K, Saito M, et al: Assessment of human brown adipose tissue density during daily ingestion of thermogenic capsinoids using near-infrared time-resolved spectroscopy. J Biomed Opt 21: 091305, 2016.

112. Kim SM, Jung YJ, Kwon ON, Cha KH, Um BH, Chung D and Pan CH: A potential commercial source of fucoxanthin extracted from the microalga Phaeodactylum tricornutum. Appl Biochem Biotechnol 166: 1843-1855, 2012. 
113. Bonet ML, Ribot J, Galmés S, Serra F and Palou A: Carotenoids and carotenoid conversion products in adipose tissue biology and obesity: Pre-clinical and human studies. Biochim Biophys Acta Mol Cell Biol Lipids 1865: 158676, 2020.

114. Hilgendorf KI, Johnson CT, Mezger A, Rice SL, Norris AM, Demeter J, Greenleaf WJ, Reiter JF, Kopinke D and Jackson PK: Omega-3 fatty acids activate ciliary FFAR4 to control adipogenesis. Cell 179: 1289-1305.e21, 2019.

115. Jiménez-Aranda A, Fernández-Vázquez G, Campos D, Tassi M, Velasco-Perez L, Tan DX, Reiter RJ and Agil A: Melatonin induces browning of inguinal white adipose tissue in Zucker diabetic fatty rats. J Pineal Res 55: 416-423, 2013.

116. Zhang Y, Goldman S, Baerga R, Zhao Y, Komatsu M and Jin S: Adipose-specific deletion of autophagy-related gene 7 (atg7) in mice reveals a role in adipogenesis. Proc Natl Acad Sci USA 106: 19860-19865, 2009

117. Martinez-Lopez N, Athonvarangkul D, Sahu S, Coletto L, Zong H, Bastie CC, Pessin JE, Schwartz GJ and Singh R: Autophagy in $\mathrm{Myf}^{+}$progenitors regulates energy and glucose homeostasis through control of brown fat and skeletal muscle development. EMBO Rep 14: 795-803, 2013.
118. Tran CM, Mukherjee S, Ye L, Frederick DW, Kissig M, Davis JG, Lamming DW, Seale P and Baur JA: Rapamycin blocks induction of the thermogenic program in white adipose tissue. Diabetes 65: 927-941, 2016.

119. Liu D, Bordicchia M, Zhang C, Fang H, Wei W, Li JL, Guilherme A, Guntur K, Czech MP and Collins S: Activation of mTORC1 is essential for $\beta$-adrenergic stimulation of adipose browning. J Clin Invest 126: 1704-1716, 2016.

120. Wada S, Neinast M, Jang C, Ibrahim YH, Lee G, Babu A, Li J, Hoshino A, Rowe GC, Rhee J, et al: The tumor suppressor FLCN mediates an alternate mTOR pathway to regulate browning of adipose tissue. Genes Dev 30: 2551-2564, 2016.

(i) () This work is licensed under a Creative Commons Attribution-NonCommercial-NoDerivatives 4.0 International (CC BY-NC-ND 4.0) License. 